\title{
Evaluation of Retinol Palmitate Treatment of Photokeratitis in Rat Eyes Exposed to Ultraviolet B Radiation
}

\author{
Mehmet Caner Filizay, ${ }^{1}$ (D) Nesrin Buyuktortop Gokcinar, ${ }^{2}$ (i) Varol Sahinturk, ${ }^{3}$ (D) Setenay Oner, ${ }^{4}$ \\ (D) Sedat Kacar, ${ }^{3}$ (1) Zafer Onaran, ${ }^{2}$ (1) Erhan Yumusak ${ }^{2}$
}

${ }^{1}$ Department of Ophthalmology, Eskisehir State Hospital, Eskisehir, Turkey

${ }^{2}$ Department of Ophthalmology, Kirikkale University Faculty of Medicine, Kirikkale, Turkey

${ }^{3}$ Department of Histology and Embryology, Eskisehir Osmangazi University Faculty of Medicine, Eskisehir, Turkey

${ }^{4}$ Department of Biostatistics, Eskisehir Osmangazi University Faculty of Medicine, Eskisehir, Turkey

\begin{abstract}
Objectives: Acute exposure to ultraviolet B radiation can cause photokeratitis. Retinol palmitate (RP) is known to have antioxidant properties and improve corneal healing. The aim of this study was to evaluate the effect of topical RP against phototoxic keratitis in rats.

Methods: A total of 14 male Wistar Albino rats were exposed to I J/cm ${ }^{2}$ dose of $31 \mathrm{I} \mathrm{nm}$ ultraviolet $B$ radiation. The subjects were then divided into 4 study groups using the right and left eye: The RP-5 group $(n=7)$ received topical $250 \mathrm{IU} / \mathrm{g}$ RP ointment and the Sham-5 group $(n=7)$ received only the vehicle base component of the ointment 5 minutes after the exposure. The RP- 120 group $(n=7)$ received topical RP and the Sham- 120 group $(n=7)$ received the vehicle alone 120 minutes after the exposure. The eyes were enucleated 24 hours after the exposure and stained with hematoxylin and eosin for histopathological examination and a terminal deoxynucleotidyl transferase dUTP nick end labeling assay to test for apoptosis. Results: There was no statistically significant difference between the mean corneal epithelial thickness of the RP- 5 group and the Sham-5 group $(p=0.369)$. Furthermore, there was no significant difference between the RP- 120 and the Sham- 120 groups $(p=0.765)$. The timing of the administration of RP resulted in no significant difference in the mean corneal epithelial thickness $(p=0.608)$. Apoptotic cell count scores were not significantly different between corneas that received RP and those who received only the vehicle $(p=0.530, p=0.107)$.

Conclusion: Topical administration of a single dose of RP was not superior to the use of the vehicle base alone in a photokeratitis model produced using I J/ $\mathrm{cm}^{2}$ of narrowband ultraviolet radiation in rats.

Keywords: Apoptosis, cornea, photokeratitis, retinol palmitate, ultraviolet, vitamin A.
\end{abstract}

\section{Introduction}

Ultraviolet radiation (UVR) is a category of the electromagnetic spectrum, found between $X$-rays and visible light. The UVR reaching earth is composed of $90 \%$ to $95 \%$ UVA and $5 \%$ to $10 \%$ UVB. UVB is potentially more hazardous than UVA because it has more energy and greater ability to damage tissue $(I, 2)$. Acute or chronic exposure to UVR may have a phototoxic effect, depending on the wavelength, energy, and duration of the exposure. Acute corneal response to pho- totoxic injury induces photokeratitis. Chronic UV exposure has been reported to be associated with climatic droplet keratopathy, pterygium, endothelial dystrophy, squamous metaplasia and carcinoma, cataract formation, and age-related macular degeneration (I, 2).

Photokeratitis, namely UV keratitis or phototoxic keratitis, is an acute photochemical response to toxic doses of UVR. Welder's arc burns and snow blindness are the best-known causes of photokeratitis. Additionally, laboratory or germicidal ultraviolet lamps, metal halide lamps,

Address for correspondence: Nesrin Buyuktortop Gokcinar, MD. Kirikkale Universitesi Tip Fakultesi, Oftalmoloji Anabilim Dali, Kirikkale, Turkey

Phone: +90 5332307412 E-mail: tortopn@kku.edu.tr

Submitted Date: February 18, 2019 Accepted Date: June 11, 2019 Available Online Date: August 05, 2019

${ }^{\circledR}$ Copyright 2019 by Beyoglu Eye Training and Research Hospital - Available online at www.beyoglueye.com OPEN ACCESS This work is licensed under a Creative Commons Attribution-NonCommercial 4.0 International License. 
and sunlamps may be related to phototoxic corneal injury (2). A latency of 6 to 12 hours between the exposure and the onset of symptoms is a characteristic feature of photokeratitis. Bilateral severe ocular pain, photophobia, decreased visual acuity are the main symptoms. Chemosis, blepharospasm, and facial erythema may also be present. Photokeratitis findings include superficial punctate keratitis, epithelial desquamation and erosion, corneal haze, and edema, and rarely, anterior chamber inflammation. Clinical symptoms usually resolve spontaneously within 72 hours (I). Histopathological examination has revealed that UVR exposure led to the separation of superficial epithelial cells and nuclear and cytoplasmic damage in the remaining attached epithelium, intracellular vacuolization and nuclear disintegration in the keratocytes, and edema between the stomal lamella due to endothelial dysfunction. Apoptosis has been observed in all layers of the cornea, depending on the dose and wavelength of UVR (I). Photokeratitis is a result of complex photochemical reactions involving many inflammatory agents, including reactive oxygen species, nuclear factor (NF)-кB, prostaglandin E2, and tumor necrosis factor-a (TNF-a) (3-6). Some antioxidant or anti-inflammatory agents, such as octreotide, lornoxicam, lactoferrin, vitamin $C$, and dietary zerumbone have been shown to have a protective effect against photokeratitis (3-6). In particular, astaxanthin, a carotenoid with strong antioxidant activity, has been shown to ameliorate corneal UVR toxicity in animal studies when applied topically or orally $(7,8)$. Provitamin A carotenoids are converted to retinol for bioavailability.

Retinol palmitate (RP) is the ester of retinol (vitamin A) and palmitic acid. Vitamin A deficiency has been reported to be associated with pre-vitamin A carotenoids (9) and increased activity of NF- $\mathrm{kB}$ and decreased activity of antioxidant enzymes, like superoxide dismutase, glutathione peroxidase, and catalase (10). The stability of RP is greater than that of vitamin $A$, which is readily inactivated by oxidation. In fact, eye drops containing RP have been demonstrated to improve antioxidant status and decrease oxidative damage (II). RP has also been reported to be effective against nheptanol and alkali corneal injury (I2). Furthermore, it has been reported to facilitate the healing of corneoscleral incisions (I3). An ophthalmic solution with RP was recently shown to be effective in the treatment of dry eye (14).

To our knowledge, the effect of RP against ultraviolet damage on the cornea has not yet been reported. Since astaxanthin has produced very effective results against photokeratitis $(7,8)$, the objective of this study was to evaluate another form of vitamin A, RP, in the same photokeratitis model. Eye ointment containing RP is commercially available and can be readily found. It is used for supportive treatment for eye inflammation and atopic corneal changes due to a lack of vitamin A. However, it is not presently used to treat welder's arc burns in current practice. This was an examination of histopathological changes and apoptotic responses to UVR and the possible beneficial effects of RP. The proposal was that if it was found to be effective, RP might be a source of a future treatment for patients with welder's arc burn. A second objective of this research was to compare the results of early and late application of RP ointments in order to estimate clinical projections.

\section{Methods}

\section{Animals}

This study was approved by Local Ethics Committee for Animal Experiments at Ankara Training and Research Hospital, Ankara, Turkey (04.04.2013/00 I3-186) and conducted according to the Association for Research in Vision and Ophthalmology Statement for the Use of Animals in Ophthalmic and Vision Research. The study was carried out on 15 male Wistar Albino rats with a weight range of 200-210 g that were purchased from Saki Yenilli Experimental Animal Laboratory (Ankara, Turkey). The animals were housed under standard laboratory conditions with constant temperature $\left(22 \pm 3^{\circ} \mathrm{C}\right)$ and a relative humidity of $65-70 \%$ with 12 -hour light and dark cycles. The animals were fed with standard chow and provided with water ad libitum.

\section{Phototoxic Keratitis Model}

Anesthesia was achieved with intraperitoneal administration of $80 \mathrm{mg} / \mathrm{kg}$ ketamine hydrochloride (Ketalar; Pfizer, Inc., NY, NY, USA) and $10 \mathrm{mg} / \mathrm{kg}$ xylazine hydrochloride (Alfazyne; Alfasan IBV, Woerden, The Netherlands). The body surface of the study subjects was covered with aluminum foil from the neck down in order to avoid UV damage. The rats were placed on the plate of a Waldmann UV $18 \mathrm{I}$ BL device (Herbert Waldmann GmbH \& Co. KG, Villingen-Schwenningen, Germany) with the ventral surface face down in order for the right and left eyes to be exposed to UV light at the same distance. The narrowband UV device was set to produce I J/cm² of energy (6). UV radiation was administered to all of the animals in our study. A no-treatment control group was created to demonstrate the effect of ultraviolet exposure. Fourteen rats were randomly assigned to 2 experimental sets according to the time interval between the irradiation and the application of ointment. Seven rats received ointment 5 minutes after the irradiation, and 7 rats received ointment 20 minutes after the irradiation. VitA-POS ointment (Ursapharm, Saarbrücken, Germany), containing 250 $\mathrm{IU} / \mathrm{g}$ RP, was administered to the right eye, and the left eye received a vehicle only, consisting of the base component 
of the ointment: a mixture of white petroleum jelly, liquid paraffin, and lanolin.

The 4 study groups were:

I. RP-5 group: Ointment containing RP was applied to the right eye of 7 rats 5 minutes after the irradiation $(n=7)$,

2. Sham-5 group: Vehicle was applied to the left eye of the same 7 rats, 5 minutes after the irradiation $(n=7)$,

3. RP-120: Ointment containing RP was applied to the right eye of the remaining 7 rats 120 minutes after the irradiation $(n=7)$,

4. Sham-120: Vehicle was applied to the left eye of the remaining 7 rats 120 minutes after the irradiation $(n=7)$.

Twenty-four hours after the irradiation, all of the rats were sacrificed by decapitation under anesthesia with intraperitoneal $50 \mathrm{mg} / \mathrm{kg}$ propofol (Abbott Laboratories, Lake Bluff, IL, USA).

\section{Hematoxylin-eosin and TUNEL Staining}

The eyes were enucleated and the corneas were fixed in 10\% formalin and embedded in paraffin. Five- $\mu$ m-thick corneal sections were prepared and stained with hematoxylin and eosin for histopathological examination and a terminal deoxynucleotidyl transferase dUTP nick end labeling (TUNEL) assay with an Apoptag Plus Peroxidase In Situ Apoptosis Detection Kit (S7I0I; MilliporeSigma, Burlington, MA, USA) to reveal apoptotic cell signaling. The specimens were examined under a light microscope Olympus $\mathrm{BH}-2$ (Olympus Corp., Tokyo, Japan) by a single histologist who was blinded to the study. Images from the light microscope were captured using a high-resolution Olympus DP 70 digital camera (Olympus Corp., Tokyo, Japan).

The corneal epithelial thickness was measured and a mean of 5 sections from the central cornea in each eye was recorded. The data are given as mean $\pm S D$. Apoptotic cell scores were evaluated in 5 sections taken from different segments of each eye. In each section, apoptotic cells were tallied under 40x magnification in 3 randomly selected areas. A semiquantitative scoring method was used to evaluate apoptotic cell density. The score ranged between 0 and 2: A score of 0 indicated no TUNEL-positive stained corneal epithelial or stromal cells in any of the sections, a score of I reflected an apoptotic cell count of less than 5 in all of the sections, and a score of 2 signified that the apoptotic cell count was equal to or more than 5 in at least I section.

\section{Statistical Analysis}

Statistical analysis was performed using IBM SPSS Statistics for Windows, Version 2I.0 (IBM Corp., Armonk, NY, USA). The Shapiro-Wilk test was used to assess the conformity of data with a normal distribution. The data are described as mean $\pm S D$. Pairwise comparisons of corneal epithelial thick- ness were analyzed using an independent samples t-test. Semiquantitative TUNEL-positive apoptotic cell gradings were analyzed with the Mann-Whitney $U$ test. Statistical significance was set at $p<0.05$.

\section{Results}

\section{Morphological Properties}

The wavelength and the dose of UVR used in our study was sufficient to induce photokeratitis in all of the rat eyes. There were significant morphological changes, particularly involving the corneal epithelium, and occasionally the stroma, in all groups (Fig. I). There were epithelial and stromal TUNELpositive stained apoptotic cells in all groups (Fig. 2). In the no-treatment control eyes, corneal epithelial irregularities, intraepithelial vacuolization, and irregular cells with pyknotic nuclei were observed in addition to TUNEL-positive stained apoptotic cells in both the epithelium and the stroma (Figs. I, $2)$. In the RP-5 group, there was evidence of corneal epithelial desquamation, irregularity, vacuolization, and pyknotic
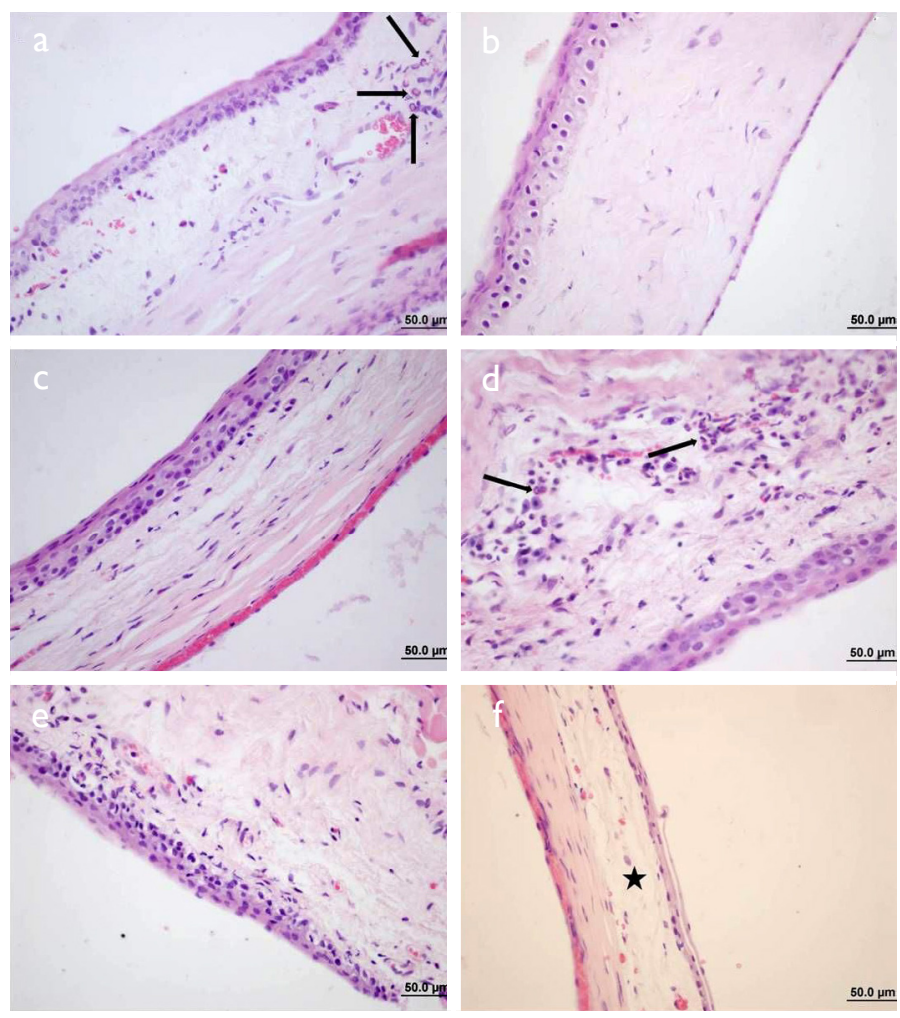

Figure I. Corneal sections, H\&E, scale bar, $50 \mu \mathrm{m}$. (a) Irregularity in the corneal epithelium and polymorphonuclear leukocyte (PMNL) cell infiltration (arrows) in the retinol palmitate (RP)-5 group, (b) epithelial irregularity in the Sham-5 group, (c) epithelial irregularity in the RPI 20 group, (d) epithelial irregularity and stromal PMNL cells (arrows) in the Sham- 120 group, (e) corneal epithelial irregularity and pyknotic nuclei in a rat that received only UV irradiation, (f) corneal epithelial irregularity, detachment, and stromal edema (star) in a rat that received only UV irradiation. 
cell nuclei. In I eye, there was an intrastromal hemorrhage and polymorphonuclear leukocyte (PMNL) infiltration. In the Sham-5 group, corneal epithelial desquamation, suprabasal clefting, vacuolization, and irregularities were seen. Intraepithelial hemorrhage was recorded in I eye. In 2 eyes, the corneal epithelium was detached from the stroma. The corneal epithelia were also irregular in the RP-I 20 group. In I eye, there was a prominent epithelial ulceration. In the Sham- I 20 group, epithelial desquamation and intra-epithelial clefting, and increased acidophilia of the superficial corneal epithelial cell cytoplasm was observed. In I eye, PMNL infiltration of the stroma was seen.

\section{Corneal Epithelial Thickness}

The corneal epithelial thickness data of the study groups are provided in Table I. There was no statistically significant differ-

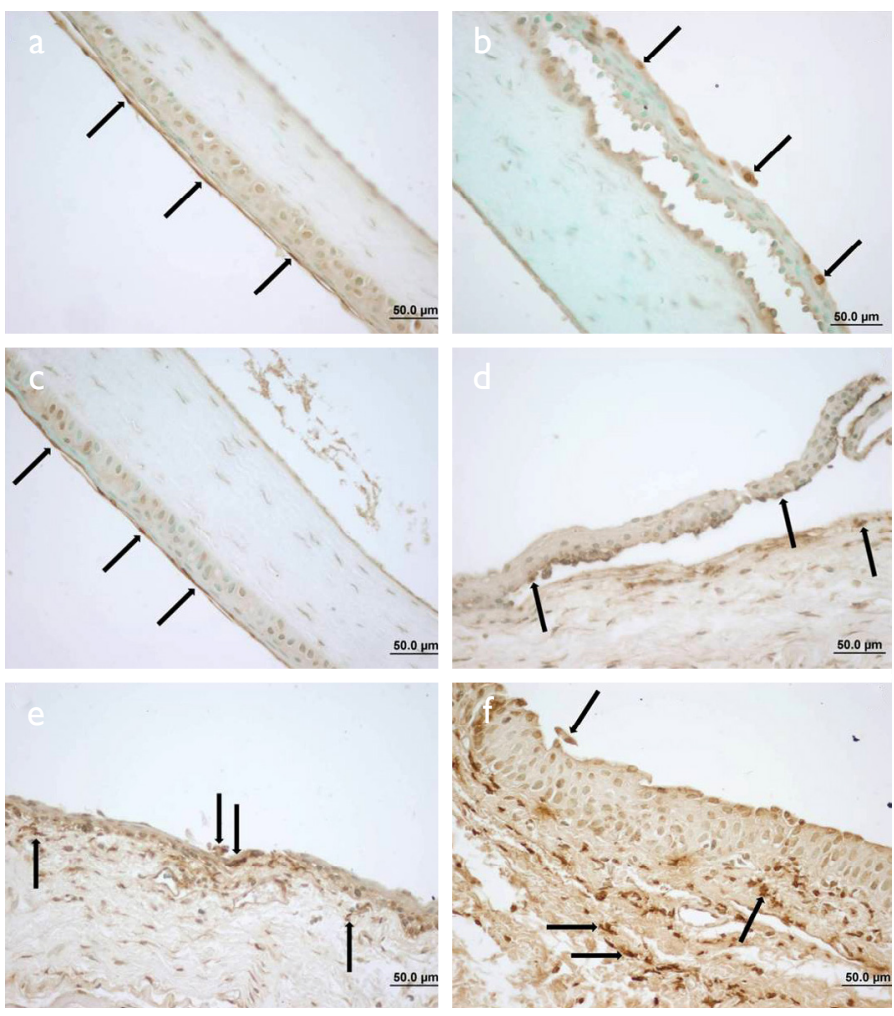

Figure 2. Corneal sections, terminal deoxynucleotidyl transferase dUTP nick end labeling staining, scale bar, $50 \mu \mathrm{m}$. (a) Positively stained apoptotic cells on the corneal epithelium in the retinol palmitate (RP)5 group (arrows), (b) epithelial irregularity, detachment, and positively stained apoptotic cells (arrows) in the epithelium of the Sham-5 group, (c) positively stained apoptotic cells on the corneal epithelium in the RP-I 20 group (arrows), (d) epithelial irregularity, detachment, and positively stained apoptotic cells (arrows) in the Sham-120 group, (e) prominent epithelial irregularity, detachment, and positively stained apoptotic cells (arrows) in the epithelium and stroma in a rat that received only UV irradiation, (f) prominent epithelial irregularity, detachment, and positively stained apoptotic cells (arrows) in the epithelium and stroma in a rat that received only UV irradiation. ence in pairwise comparisons of mean corneal epithelial thickness values. The $p$ values of pairwise comparisons are illustrated in Figure 3. RP use was not superior to the vehicle alone in terms of preserving epithelial thickness when applied 5 minutes or 120 minutes after the irradiation. There was no significant difference in the early ( 5 minutes) or late ( 120 minutes) application of RP in terms of corneal epithelial protection.

\section{TUNEL Staining}

The apoptotic cell scores of the study groups observed in TUNEL staining are given in Table 2. There was no statistically significant difference in the pairwise comparisons of apoptotic cell scores in the study groups. RP was not superior to the vehicle when applied 5 minutes or 120 minutes after the exposure to irradiation. Additionally, there was no statistically significant difference between the apoptotic cell scores of the RP- 5 and RP- 120 groups according to the timing of agents delivered. The $p$ values of the pairwise comparisons can be seen in Figure 4 .

\section{Discussion}

UVR has been shown to damage all corneal layers. In the epithelium, it has caused exfoliation of superficial layers, ulceration, and PMNL infiltration. In the stroma, keratocyte damage with intracellular vacuoles, fragmentation, and nuclear disintegration, as well as edema between the stromal layers and around the keratocytes have been reported. Endothelial dysfunction, pleomorphism, and rosette formation have been observed (15-17). UVR can also affect the lens and the retina, depending on the wavelength, dose, and duration of the radiation exposure (I7).

The corneal epithelium is the layer that is most sensitive

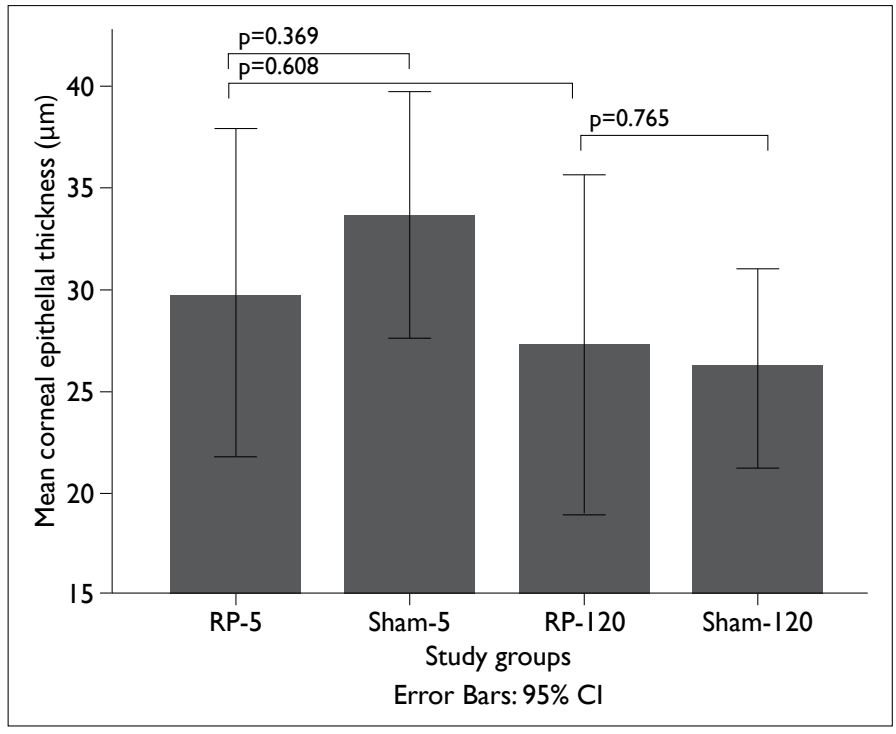

Figure 3. The mean corneal epithelial thickness in the study groups. The $\mathrm{p}$-values belong to the independent samples t-test. 
Table I. Corneal epithelial thickness in the study groups

\begin{tabular}{|c|c|c|c|c|}
\hline \multirow[t]{2}{*}{ Groups } & \multicolumn{4}{|c|}{ Corneal epithelial thickness ( $\mu \mathrm{m})$} \\
\hline & Mean \pm SD & Median & Minimum-Maximum & $\mathbf{p}$ \\
\hline $\operatorname{RP}-5(n=7)$ & $29.82 \pm 8.75$ & 27.08 & $(21.07-44.91)$ & \\
\hline Sham-5 $(n=7)$ & $33.68 \pm 6.54$ & 34.14 & $(26.58-44.10)$ & $0.369^{\circ}$ \\
\hline RP-I $20(n=7)$ & $27.32 \pm 9.00$ & 22.53 & $(19.40-42.97)$ & $0.608^{a}$ \\
\hline Sham- $120(n=7)$ & $26.12 \pm 5.28$ & 25.04 & $(17.90-32.02)$ & $0.765^{\beta}$ \\
\hline
\end{tabular}

a: $\mathrm{p}$ value when compared with RP-5 group; ${ }^{\beta}: \mathrm{p}$ value when compared with RP-I 20 group. Independent samples t-test.

Table 2. TUNEL staining results in the study groups

\begin{tabular}{|c|c|c|c|c|}
\hline \multirow[t]{2}{*}{ Groups } & \multicolumn{4}{|c|}{ TUNEL staining scores } \\
\hline & Mean \pm SD & Median & Minimum-Maximum & $\mathbf{p}$ \\
\hline RP-5 $(n=7)$ & $1.7 I \pm 0.48$ & 2 & $(I-2)$ & \\
\hline Sham-5 $(n=7)$ & $1.86 \pm 0.37$ & 2 & $(I-2)$ & $0.530^{\circ}$ \\
\hline$R P-120(n=7)$ & $1.43 \pm 0.53$ & I & $(I-2)$ & $0.298^{a}$ \\
\hline Sham-I $20(n=7)$ & $1.86 \pm 0.37$ & 2 & $(I-2)$ & $0.107^{\beta}$ \\
\hline
\end{tabular}

a: $\mathrm{p}$ value when compared with RP-5 group, ${ }^{\text {B: }}$ : v value when compared with RP-I 20 group. Mann-Whitney $U$ test. TUNEL: terminal deoxynucleotidyl transferase dUTP nick end labeling.

to UVR. It has been demonstrated that the corneal stroma and the endothelium were affected by wavelengths above $290 \mathrm{~nm}$ (17). UVR with a wavelength of $310 \mathrm{~nm}$ was determined to have induced apoptosis in all of the corneal layers, including the epithelium, stroma, and endothelium (15). Based on the literature, a narrowband UVB device with a

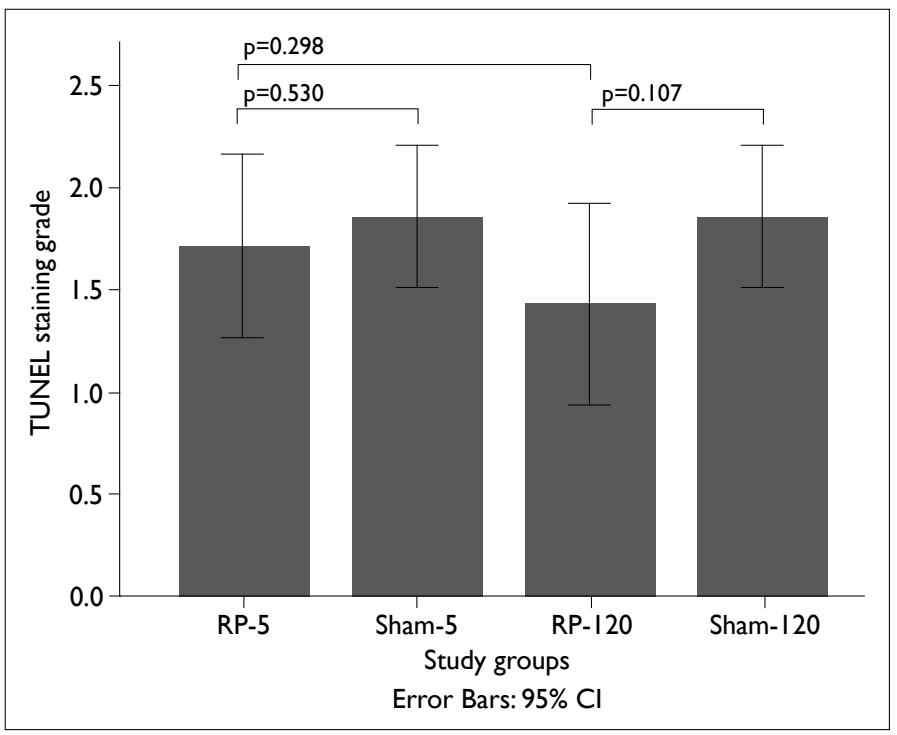

Figure 4. HbAlc: glycated hemoglobin; NPDR: non-proliferative diabetic retinopathy; PDR: proliferative diabetic retinopathy.

${ }^{*} \mathrm{p}<0.05$ in PDR vs. control; $\mathrm{p}<0.05$ in severe NPDR vs. control; $\mathrm{p}>0.05$ in PDR vs. severe NPDR. peak wavelength of $31 \mathrm{I} \mathrm{nm}$ was used in our study to produce a phototoxic keratitis model that would affect all layers of the cornea $(3,4,17)$. Earlier research has established that $1 \mathrm{~J} / \mathrm{cm}^{2}$ and $1.2 \mathrm{~J} / \mathrm{cm}^{2}$ of UVR with $310-315 \mathrm{~nm}$ wavelengths induced photokeratitis in rats and mice $(3,4)$. A phototoxic corneal injury model was established in our study using I $\mathrm{J} / \mathrm{cm}^{2} 3 \mathrm{II} \mathrm{nm}$ of UVB radiation in all eyes, similar to that described in published reports. The postulated mechanisms of UVR-induced damage include cumulative DNA damage, changes in membrane transport and permeability, and damage by reactive oxygen species, which induce the production of proinflammatory molecules (18). UVR has been reported to increase the production of interleukin (IL)-I, IL-6, IL-8, and TNF-a in human corneal stromal cells, and to activate the translocation of NF-kB, which induces the transcription of proinflammatory cytokines, adhesion molecules, and other mediators involved in inflammation, cell proliferation, and apoptosis $(18,19)$.

Some antioxidant agents have been reported to have a protective effect against photokeratitis (3-8). Intraperitoneal treatment with lornoxicam, a non-steroidal anti-inflammatory agent and a potent COX inhibitor, suppressed UVB-induced NF- $\mathrm{kB}$ activation and TNFa production and significantly improved corneal haze and histopathological degenerative findings in the mouse cornea. Untreated corneas were severely damaged, resulting in epithelial cell loss, swollen basal cells, hydropic stroma, and loss of keratocytes 
after exposure to $1.2 \mathrm{~J} / \mathrm{cm}^{2}$ of UVR with a wavelength of approximately $310 \mathrm{~nm}$ (3). In another morphometrical and immunohistochemical study of the effects of lornoxicam, I.2 $\mathrm{J} / \mathrm{cm}^{2}$ narrowband UVB exposure resulted in epithelial thinning with cellular degeneration, exfoliation, and metaplasia, as well as a loss in stromal area and keratocyte count in rat corneas. The mean epithelial thickness was $4 \mathrm{I} \mu \mathrm{m}$ in control eyes with no UV exposure or treatment, $25 \mu \mathrm{m}$ in the UVirradiated group without any treatment, and approximately $38 \mu \mathrm{m}$ in the group that received intraperitoneal injections of $0.4 \mathrm{mg} / \mathrm{kg} /$ day lornoxicam for I week after the irradiation (20).

Dietary alpha-lipoic acid has been reported to prevent UVB-induced corneal damage by reducing malondialdehyde accumulation, NF-кB, COX-2, TNF-a, IL-6, and matrix metalloproteinase 9 activity, in a mouse photokeratitis model produced by $0.72 \mathrm{~J} / \mathrm{cm}^{2}$ of UVB irradiation with a peak wavelength of $312 \mathrm{~nm}(21)$.

In another study, astaxanthin, a xanthophyll carotenoid of predominantly marine origin with potent antioxidant and anti-inflammatory effects, was found to ameliorate UV-induced photokeratitis in mice by reducing NF-kB expression in the corneal epithelia. In that study, $400 \mathrm{~mJ} / \mathrm{cm}^{2}$ of UVB radiation led to apoptosis and thinning of the corneal epithelium. Administration of $1 \mathrm{mg} / \mathrm{mL}$ of astaxanthin 5 minutes after the irradiation resulted in a thicker mean corneal epithelium than that measured in the fellow eyes, which received vehicle alone. The epithelium was morphologically better preserved and there were significantly fewer TUNEL-positive cells in the corneas treated with astaxanthin than those that received only the vehicle (7).

Consistent with the literature, in our study, 3 I I nm UVR at a dose of $\mathrm{IJ} / \mathrm{cm}^{2}$ caused desquamation in the superficial epithelial layers, irregularity, vacuolization, and granulocytic infiltration in both the epithelium and the stroma. There was no statistically significant difference between the treatment and sham groups with treatment at 5 minutes or 120 minutes after the irradiation. Immediate administration of RP was not significantly better than the delayed administration ( 5 minutes versus 120 minutes) in terms of the mean epithelial thickness. In contrast to astaxanthin, RP did not demonstrate a significant protective effect against UV-related apoptosis in our study. RP has been reported to have anti-oxidant effects, but it may not be as potent as astaxanthin (10-12, 22).

RP has been reported to be found in lacrimal gland cells (23) and it has been used in the treatment of ocular surface diseases, such as dry eye and superior limbic keratoconjunctivitis, and also after corneal refractive surgery (I I, I4, 23, 24). In a phase II clinical trial, vitamin A palmitate ophthalmic solution was reported to be safe and effective for the treatment of patients with dry eye (14). It promotes mucin production and healing of the keratoconjunctival epithelium (12). RP has been shown to inhibit the decrease of conjunctival goblet cells after corneal allograft rejection in rats (25). It has also been reported to improve antioxidant status and restore enzymatic and nonenzymatic defenses, as well as decrease protein oxidative damage (10). However, in another study comparing the free radical scavenging properties of RP with retinyl ascorbate, ascorbic acid, retinoic acid, and retinol, RP had no significant antioxidant properties, whereas other retinoids demonstrated some strength. Retinyl ascorbate had the greatest potency (22).

To our knowledge, there is no information in the literature about the usage of vitamin A palmitate against UV injury on the cornea. Our research indicated that topical application of single-dose RP was insufficient to prevent apopto-

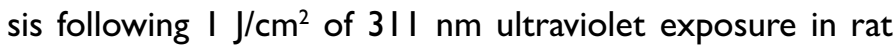
corneal tissue. Histopathologically, RP was not superior to vehicle alone in terms of corneal epithelial thickness.

The primary limitation of our study is the lack of a blank control group. The mean corneal epithelial thickness of nonirradiated, non-treated control rats has been well studied and reported to be approximately $40 \mu \mathrm{m}$. Photokeratitis was established in all of our study groups. All of the corneas had some degree of UVR injury. In order to sacrifice as few animals as possible, we only included treatment and sham groups and evaluated the possible superiority of RP ointment to a vehicle alone and against the photokeratitis model.

In conclusion, RP may not be superior to vehicle in terms of preventing phototoxic corneal injury. Further studies using different antioxidant agents to prevent and treat photokeratitis may be useful for the quick recovery of patients exposed to toxic doses of UVR.

\section{Disclosures}

Ethics Committee Approval: This study was approved by Local Ethics Committee for Animal Experiments at Ankara Training and Research Hospital, Ankara, Turkey (04.04.2013/00 I3-186) and conducted according to the Association for Research in Vision and Ophthalmology Statement for the Use of Animals in Ophthalmic and Vision Research.

Peer-review: Externally peer-reviewed.

Conflict of Interest: None declared.

Authorship Contributions: Involved in design and conduct of the study (NBG, MCF, VS, SO, ZO); preparation and review of the study (NBG, MCF, VS, SK, Z.O, EY); data collection (MCF, VS, SK, NBG); and statistical analysis (SO, VS, NBG).

\section{References}

I. Hamill MB. Corneal trauma. In: Krachmer JH, Mannis MJ, Holland EJ, editors. Cornea. 3rd ed. Saint Louis: Mosby Elsevier; 2010. P. II76-8. 
2. Jacobs DS. Photokeratitis. In: Trobe J, Wilterdink J, editors. UpToDate. Available at: https://021081217-y-https-www-uptodate-com.proxy.kirikkale-elibrary.com/contents/ photokeratitis. Accessed Aug 10, 2018.

3. Yin J, Huang Z, Wu B, Shi Y, Cao C, Lu Y. Lornoxicam protects mouse cornea from UVB-induced damage via inhibition of NF\{kappa\}B activation. Br J Ophthalmol 2008;92:562-8. [CrossRef]

4. Fujihara T, Nagano T, Endo K, Nakamura M, Nakata K. Lactoferrin protects against UV-B irradiation-induced corneal epithelial damage in rats. Cornea 2000;19:207-I I. [CrossRef]

5. Chen BY, Lin DP, Wu CY, Teng MC, Sun CY, Tsai YT, et al. Dietary zerumbone prevents mouse cornea from UVB-induced photokeratitis through inhibition of NF-kB, iNOS, and TNF-a expression and reduction of MDA accumulation. Mol Vis 201 I; 17:854-63.

6. Demir U, Demir T, Akpolat $\mathrm{N}$. The effect of octreotide against oxidative damage in photosensitized conjunctiva and cornea of rabbits. Doc Ophthalmol 2005; I 10:193-20I. [CrossRef]

7. Lennikov A, Kitaichi N, Fukase R, Murata M, Noda K, Ando R, et al. Amelioration of ultraviolet-induced photokeratitis in mice treated with astaxanthin eye drops. Mol Vis 20I2; I8:455-64.

8. Harada F, Morikawa T, Lennikov A, Mukwaya A, Schaupper $M$, Uehara $O$, et al. Protective Effects of Oral Astaxanthin Nanopowder against Ultraviolet-Induced Photokeratitis in Mice. Oxid Med Cell Longev 2017;2017:1956104. [CrossRef]

9. Kontos A, Kayhanian H, El-Khouly F, Gillmore R. Night blindness due to vitamin $A$ deficiency associated with resected adenocarcinoma of the pancreas. Int J Ophthalmol 20I5;8:206-7.

10. Behr GA, Schnorr CE, Moreira JC. Increased blood oxidative stress in experimental menopause rat model: the effects of vitamin A low-dose supplementation upon antioxidant status in bilateral ovariectomized rats. Fundam Clin Pharmacol 20I2;26:235-49. [CrossRef]

I I. Ohashi Y, Watanabe H, Kinoshita S, Hosotani H, Umemoto M, Manabe R. Vitamin A eyedrops for superior limbic keratoconjunctivitis. Am J Ophthalmol 1988;105:523-7. [CrossRef]

12. Toshida H, Odaka A, Koike D, Murakami A. Effect of retinol palmitate eye drops on experimental keratoconjunctival epithelial damage induced by $n$-heptanol in rabbit. Curr Eye Res 2008 Jan;33:13-8. [CrossRef]

I3. Kastl PR, Rosenthal WN, Batlle I, Karcioglu Z. Topical vitamin A ointment increases healing of cataract incisions. Ann Ophthalmol 1987;19:175-7.
14. Toshida H, Funaki T, Ono K, Tabuchi N, Watanabe S, Seki T, et al. Efficacy and safety of retinol palmitate ophthalmic solution in the treatment of dry eye: a Japanese Phase II clinical trial. Drug Des Devel Ther 2017;1 I:I87I-9. [CrossRef]

15. Podskochy A, Gan L, Fagerholm P. Apoptosis in UV-exposed rabbit corneas. Cornea 2000;19:99-103. [CrossRef]

16. Riley MV, Susan S, Peters MI, Schwartz CA. The effects of UV-B irradiation on the corneal endothelium. Curr Eye Res 1987;6:1021-33. [CrossRef]

17. Pitts DG, Cullen AP, Hacker PD. Ocular effects of ultraviolet radiation from 295 to $365 \mathrm{~nm}$. Invest Ophthalmol Vis Sci 1977;16:932-9.

18. Kennedy M, Kim KH, Harten B, Brown J, Planck S, Meshul C, et al. Ultraviolet irradiation induces the production of multiple cytokines by human corneal cells. Invest Ophthalmol Vis Sci 1997;38:2483-9|.

19. Lee DH, Kim JK, Joo CK. Translocation of nuclear factor-kap$\mathrm{paB}$ on corneal epithelial cells induced by ultraviolet $\mathrm{B}$ irradiation. Ophthalmic Res 2005;37:83-8. [CrossRef]

20. Mahmoud BL, Shady AM, El Meleegy UAG, Soliman, MA. Effects of Ultraviolet B Radiation on the Cornea of Adult Male Albino Rats and the Possible Role of Lornoxicam: A Histological, Immunohistochemical and Morphometrical Study. Egypt. J Histol 2010;33:156-67. [CrossRef]

2I. Chen BY, Lin DP, Chang LS, Huang TP, Liu HJ, Luk CP, et al. Dietary a-lipoic acid prevents UVB-induced corneal and conjunctival degeneration through multiple effects. Invest Ophthalmol Vis Sci 2013;54:6757-66. [CrossRef]

22. Abdulmajed K, McGuigan C, Heard CM. Topical delivery of retinyl ascorbate: 4 . Comparative anti-oxidant activity towards DPPH. Free Radic Res 2005;39:49I-8. [CrossRef]

23. Odaka A, Toshida H, Ohta T, Tabuchi N, Koike D, Suto C, et al. Efficacy of retinol palmitate eye drops for dry eye in rabbits with lacrimal gland resection. Clin Ophthalmol 2012;6:158593. [CrossRef]

24. Vetrugno M, Maino A, Cardia G, Quaranta GM, Cardia L. A randomised, double masked, clinical trial of high dose vitamin $A$ and vitamin $E$ supplementation after photorefractive keratectomy. Br J Ophthalmol 200I;85:537-9. [CrossRef]

25. Zhang Y, Huang WR, Zhang S, Liu YP. Effect of vitamin A on the conjunctival goblet cells of the rat after corneal transplantation. Int J Ophthalmol 2008; I:109-12. 